\title{
Ist Evidenz der allein entscheidende Maßstab?
}

\author{
Jürgen Ferdinand Riemann
}

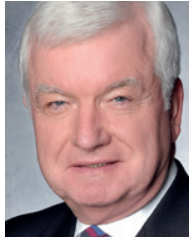

Prof. Dr. Jürgen F. Riemann ist Vorstandsvorsitzender der Stiftung LebensBlicke und em. Direktor der Med. Klinik C am Klinikum Ludwigshafen riemannj@garps.de

Interessenkonflikt

Der Autor gibt an, dass kein Interessenkonflikt besteht.

DOI 10.1055/s-0042-105303

Dtsch Med Wochenschr 2016; 141: 732

(c) Georg Thieme Verlag KG . Stuttgart · New York .

ISSN 0012-0472
Krebsvorsorge am Scheideweg I Mit dem 2013 beschlossenen Krebsfrüherkennungs- und -registergesetz (KFRG), das noch 2016 umgesetzt werden soll, steht ein Paradigmenwechsel in der Krebsfrüherkennung bevor:

- von einem opportunistischen Screening-Angebot

- zu einem organisierten, bundesweiten persönlichen Einladungsverfahren.

Für die Darmkrebsfrüherkennung sollen hierbei immunologische Stuhltests die bisherigen Gujakbasierten Tests ablösen: Neben der einfacheren Handhabung haben sie den Vorteil einer deutlich höheren Sensitivität (richtig positiv) bei mindestens genauso guter Spezifität (richtig negativ).

Quantitativer Test: bessere Evidenz I Der Gemeinsame Bundesausschuss muss bei den immunologischen Tests nun entscheiden zwischen

- einem quantitativen und

- einem qualitativen Test (Schnelltest).

Die Evidenz quantitativer Tests ist vielfach untersucht und ihre Zuverlässigkeit geprüft. Zu qualitativen Tests gibt es ebenfalls wissenschaftliche Belege, sie haben aber durchaus Schwächen. Haug und Becker (s. S. 729) favorisieren, wie bereits Experten in Gremien zur Vorbereitung der Einführung, das qualitätsgesicherte Screening durch quantitative immunochemische Stuhltests - aus meiner Sicht möglicherweise zu einseitig.

Referenzwerte fraglich | Die Qualität quantitativer Tests ist unbestritten; nicht klar ist aber nach wie vor, gegen welchen unabhängigen Referenzwert diese Tests geprüft worden sind und in Zukunft geprüft werden sollten, wenn es zu Ringversuchen kommt. Gibt es einen solchen unabhängigen Referenzwert überhaupt, und wenn ja, welche unabhängige Institution hat ihn erarbeitet?

Die Logistik verändert sich grundlegend | Mit der Einführung quantitativer Tests kommen zunehmend Laborärzte ins Spiel; die Testung soll sich sogar nur auf wenige Labors in Deutschland konzentrieren, um die Qualitätssicherung noch besser zu machen. Kann und wird die Veränderung der gesamten bisherigen Logistik wirklich zu einer Verbesserung der Inanspruchnahme gerade niedrigschwelliger Tests führen, die eigentlich doch auch neben der informierten Entscheidung erwartet wird?

Niedrigschwellige Angebote in Gefahr I Triebfedern der Früherkennung waren bisher niedrig- schwellige Stuhltests bei Hausärzten, Gynäkologen und Gastroenterologen, die derzeit ca. 4. Millionen Testbriefchen pro Jahr ausgeben. Werden sie nicht frustriert sein, wenn solche Tests künftig über Laborärzte gemacht und auch abgerechnet werden, ihnen aber nur noch die Aufklärung bzw. der finale Kommentar zugedacht wird? Womöglich beteiligen sich viele Ärzte dann gar nicht mehr am Screening.

Dokumentation und Evaluation | Wissenschaftler haben vorgeschlagen, den Koloskopie-Erfassungsbogen geringfügig zu ändern und alle eingesetzten Stuhltests lückenlos zu dokumentieren (Dtsch Arztebl 2014; 111: A-842). Dies könnte zu einer vernünftigen, qualitätsgesicherten und berechenbaren Analyse mit Evaluation führen, die keiner aufwendigen Laboruntersuchungen bedarf und damit auch kostengünstiger wäre.

Vorteil Arzt-Patienten-Beziehung I Eine entscheidende Rolle dürfte auch das strukturierte Beratungsgespräch durch den Arzt spielen, wie es im Nationalen Krebsplan gefordert wird:

- mit der Ausgabe eines niedrigschwelligen Stuhltests und

- der Möglichkeit der direkten Untersuchung und der unmittelbaren Ergebnismitteilung an den Patienten (z. B. Point-of-Care-Tests).

Man muss sich immer fragen,

- wie eine möglichst umfassende informierte Entscheidung erzielt werden kann,

- aber auch, wie ein Massenscreening mit dem geringst nötigen Aufwand bei höchstmöglichem Nutzen erreichbar ist.

Ergebnisqualität vs. Inanspruchnahme | Die Verfechter der Einführung quantitativer immunochemischer Stuhltests haben von der rein fachlichen Seite her bezogen auf die Ergebnisqualtät durchaus recht. Was nützen aber höchstmögliche Evidenz und beste Qualität, wenn durch andere Stellschrauben die Inanspruchnahme gerade der niedrigschwelligen Tests absinken und die Rolle der bisher beteiligten Ärzte in der Darmkrebsvorsorge und -Früherkennung reduziert würde? Damit wäre nichts gewonnen! Eine Vorstellung, die für engagierte Verfechter der Darmkrebsvorsorge und -Früherkennung hoffentlich nicht real wird, falls sich die Vertreter der Krankenkassen und der Kassenärztlichen Bundesvereinigung allein auf den quantitativen Test festlegen sollten. 